\title{
Neural Encoding of Objects Relevant for Navigation and Resting State Correlations with Navigational Ability
}

\author{
Joost Wegman and Gabriele Janzen
}

\begin{abstract}
Objects along a route can help us to successfully navigate through our surroundings. Previous neuroimaging research has shown that the parahippocampal gyrus (PHG) distinguishes between objects that were previously encountered at navigationally relevant locations (decision points) and irrelevant locations (nondecision points) during simple object recognition. This study aimed at unraveling how this neural marking of objects relevant for navigation is established during learning and postlearning rest. Twenty-four participants were scanned using fMRI while they were viewing a route through a virtual environment. Eye movements were measured, and brain responses were time-locked to viewing each object. The PHG showed increased responses to decision point objects compared with nondecision point objects during route learning. We compared functional connectivity be-
\end{abstract}

\section{INTRODUCTION}

To successfully navigate in the world, humans memorize information about their environment, such as the maplike spatial layout and the locations of objects. Imaging studies using virtual environments explored by participants from a first-person perspective showed that the encoding of topographical spatial knowledge invoked the hippocampus (Doeller, King, \& Burgess, 2008; Shelton \& Gabrieli, 2002; Maguire, Frackowiak, \& Frith, 1996) and the parahippocampal gyrus (PHG; Weniger et al., 2010). This latter region, which shows sensitivity to the presentation of visual scenes (Epstein, 2008; Epstein \& Kanwisher, 1998), is also involved in the successful encoding of spatial information based on landmarks (Baumann, Chan, \& Mattingley, 2010; Maguire, Frith, Burgess, Donnett, \& O'Keefe, 1998). Possibly related to a role in landmarkbased encoding, objects in isolation activate the PHG according to their associated spatial context. Compared with viewing objects that participants previously encountered at locations irrelevant for navigation (objects at nondecision points), the PHG was found to be more active when participants looked at objects that were previously encountered in a virtual or real environment at a decision point, that is, a crossing (Schinazi \& Epstein, 2010; Janzen, Jansen, \& van Turennout, 2008; Janzen, Wagensveld, \&

Radboud University Nijmegen tween the PHG and the rest of the brain in a resting state scan postlearning with such a scan prelearning. Results show that functional connectivity between the PHG and the hippocampus is positively related to participants' self-reported navigational ability. On the other hand, connectivity with the caudate nucleus correlated negatively with navigational ability. These results are in line with a distinction between egocentric and allocentric spatial representations in the caudate nucleus and the hippocampus, respectively. Our results thus suggest a relation between navigational ability and a neural preference for a specific type of spatial representation. Together, these results show that the PHG is immediately involved in the encoding of navigationally relevant object information. Furthermore, they provide insight into the neural correlates of individual differences in spatial ability.

van Turennout, 2007; Janzen \& van Turennout, 2004). This decision point effect was also observed for objects that participants had forgotten during an old/new recognition task, suggesting that the neural marking of the navigational relevance occurs as an automatic process, independent of explicit memory (Janzen \& van Turennout, 2004). These findings strongly suggest that the PHG marks objects according to their navigational relevance, which can be observed in the absence of their associated spatial context. However, the mechanisms that play a role in the initial establishment of these neural markers are unknown.

In the current fMRI study, we first investigated whether the decision point effect is immediately established when participants view objects at decision points or whether this happens at a later point in time. Participants learned routes through virtual environments, in which objects were placed at decision or nondecision points. Routes were learned inside the scanner while the participant's gaze location was monitored. Information about the first gaze directed to an object was used to determine the onset of the trial in the data analysis. We were interested in the difference in neural activity between the encoding of objects at decision points and the encoding of those at nondecision points. We predicted that the differential encoding for navigational relevance is immediately established, meaning the PHG would show higher activations for objects at decision points than for objects at nondecision points upon the first moment they are encountered during the exploration 
of a novel environment. Participants also performed a recognition test in the scanner, which allowed us to compare the regions involved in both encoding and retrieval of navigationally relevant information. We also expected the PHG to be more sensitive to decision point objects compared with nondecision point objects during simple object recognition (Janzen et al., 2007; Janzen \& van Turennout, 2004).

Second, we investigated how spatial environment learning affects the functional connectivity in the resting brain. Before and after the learning of the virtual environment, we obtained resting state functional connectivity scans (Fox \& Raichle, 2007; Biswal, Yetkin, Haughton, \& Hyde, 1995). By using the bilateral PHG as a seed region, we investigated how functional correlations between this brain region coding for navigational relevance and the rest of the brain change as a function of spatial learning. After the scanning session, participants performed a source memory task on objects seen previously in the virtual environment and answered standardized questions about their navigational skills on the Santa Barbara Sense of Direction scale (SBSOD; Hegarty, Richardson, Montello, \& Lovelace, 2002). Functional connectivity can be induced by cognitive state (Waites, Stanislavsky, Abbott, \& Jackson, 2005) and prior tasks (Hasson, Nusbaum, \& Small, 2009). The ongoing activity in resting state networks after performing tasks is thought to reflect off-line reprocessing of prior experiences to support memory formation (Tambini, Ketz, \& Davachi, 2010; Miall \& Robertson, 2006). For example, increases in the resting state functional connectivity of a test group were shown in a motor learning task compared with controls that simply performed motor tasks without learning (Albert, Robertson, \& Miall, 2009). Additional support for reprocessing that supports memory formation comes from a study that found increased connectivity between the hippocampus and the medial pFC during encoding as well as postencoding rest for a group that did not have a prior schema to facilitate learning (van Kesteren, Fernández, Norris, \& Hermans, 2010). Therefore, by looking at the learninginduced functional resting connectivity changes, we set out to identify the connections with the PHG that correlated with self-reported navigational skills and connections that predicted performance on later memory tests. People can navigate according to an egocentric (body-centered) or an allocentric (world-centered) strategy. These strategies rely on the caudate nucleus and the hippocampus, respectively (Doeller et al., 2008; Hartley, Maguire, Spiers, \& Burgess, 2003; Iaria, Petrides, Dagher, Pike, \& Bohbot, 2003). Therefore, we specifically analyzed the connectivity between the PHG and these regions for correlations with the navigational skills of participants.

\section{METHODS}

\section{Participants}

Twenty-four neurologically healthy participants (12 women, mean age $=20.3$ years, range $=18-24$ years) with normal or corrected-to-normal vision participated in our experiment. All participants were right-handed according to selfreport. Participants received a monetary reward or course credits for their participation, and all gave informed consent according to institutional guidelines of the local ethics committee (CMO region Arnhem-Nijmegen, The Netherlands).

\section{Stimulus Material and Procedures}

The experiment in the scanner comprised four parts. The first part consisted of a resting state scan lasting $7.5 \mathrm{~min}$, during which the light in the scanner room was dimmed and participants were instructed to keep their eyes open and think of nothing in particular. Such an functional connectivity MRI scan (Fox \& Raichle, 2007; Biswal et al., $1995)$ is used to detect correlations in spontaneous BOLD signal oscillations at a low frequency $(<0.1 \mathrm{~Hz})$ during a period in which the subject is not performing a task.

The prelearning resting state scan was followed by a route learning session of virtual environments in which routes were presented as video segments. During this route learning session, objects appeared on posters along the route (Figure 1). Empty trials, in which no object was presented, were included to allow us to control for the visual difference between decision and nondecision points. Eye movements were measured during the entire route learning session and brain responses were time-locked to the initial viewing of each object.

Participants were given the following standardized written instruction for the route learning session: "You are applying for a job in a museum that exhibits belongings of famous people. You will be guided through four sections of the museum, which can be distinguished from each other by the color of the floor. The exhibits are placed on posters that are hanging from the ceiling. Importantly, after training, you should be able to guide a children's tour through the museum. Therefore, while you are watching the film sequences pay special attention to toys and other things interesting for children."

The tour was given in the form of videos that showed a route through a virtual environment from a first-person perspective. Participants were instructed to learn the route and objects along the route and to pay special attention to objects interesting for children. The instruction to pay special attention to a specific object category was included to control for possible differences of attention between decision and nondecision points. The four mazes were shown in separate film sequences of $7 \mathrm{~min}$ each. The presentation order of the videos was counterbalanced over participants. Objects appeared on posters and could be placed at decision points (intersections; D objects) or nondecision points (simple turns; ND objects; Figure 1). Each section of the museum consisted of nine attended (toy) objects and nine nonattended (nontoy) objects placed at decision points, nine toy and nine nontoy objects placed at nondecision points, nine empty decision points and nine empty nondecision points. In each experimental condition, the 
Figure 1. Virtual museum and experimental protocol. (A) Virtual museum and conditions during the route learning session. Part of the virtual museum layout depicted from an aerial perspective (left). The arrow indicates the route taken. Corresponding to the route taken, examples of scenes that participants viewed during the route learning session of the experiment are shown (right). Trial types are the following: (a) unattended object at a decision point, (b) attended object at a nondecision point, (c) empty nondecision point trial (ND empty), and (d) empty decision point trial (D empty). Information about the first gaze directed to the object was used to determine the onset of the trial in the data analysis (see Methods). (B) Timeline of recognition and maze tests. During the recognition task in the scanner, participants were presented with objects they had seen previously in the museum, randomly intermixed with new distractor objects. They indicated with a button press whether they had seen the object before in the museum or not. Outside the scanner, participants performed a source memory test, in which they were presented with objects they had previously seen in the museum. With a button press they indicated in which of the four sections of the museum they had seen the object before.
A

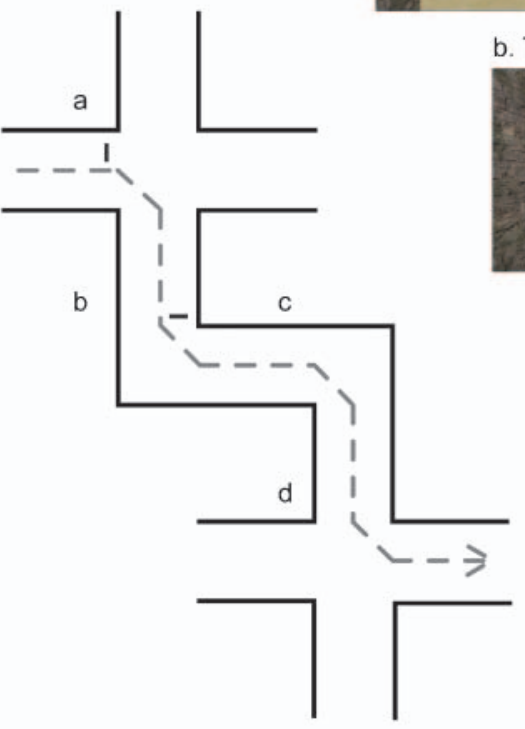

a. Nontoy object at Decision point

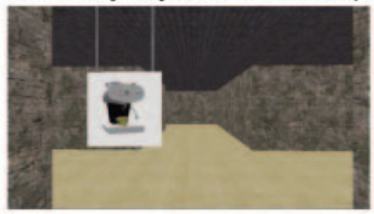

b. Toy object at Nondecision point

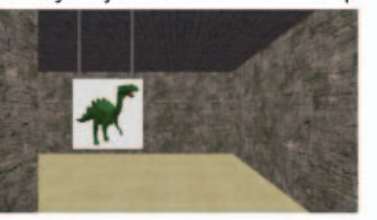

c. Nondecision point - baseline

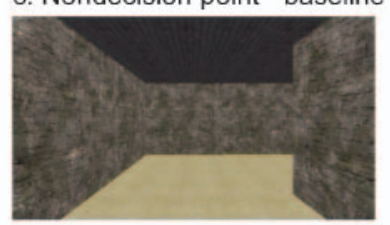

d. Decision point - baseline

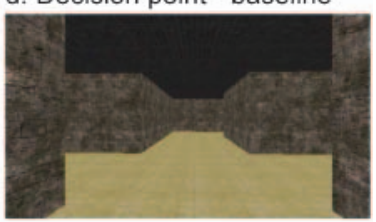

B Fixation

Object $-500 \mathrm{msec}$

Response

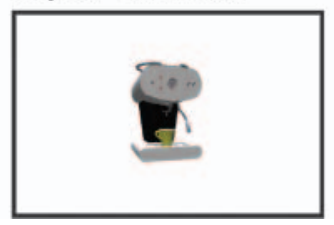

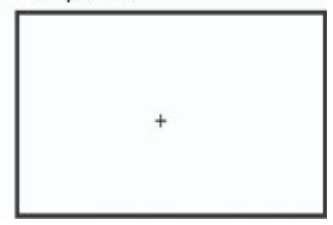

number of left and right turns made after each trial was counterbalanced over all sections of the museum. In the object trials, the number of posters hanging on the left side and the right side with respect to the viewpoint was counterbalanced over all sections of the museum. A total number of 144 stimuli were presented during the route learning session of the experiment.

The virtual environment from which the videos were recorded was created using the video game Unreal Tournament 3 (Epic Games). Objects appeared on white posters that were hanging from the ceilings of the corridors. Objects appeared as images on a white background at the moment the viewpoint was at a fixed distance from the poster in the virtual world. Although the viewpoint moved past the poster, the objects on the posters remained visible for $3 \mathrm{sec}$ on average. The viewpoint was placed at a simulated $1.70 \mathrm{~m}$ above the ground, at the same height as the center of the posters. Corridors were $4.5 \mathrm{~m}$ wide and $3.4 \mathrm{~m}$ high. The length of the corridors within a condition was on average $6.7 \mathrm{~m}$, jittered over different trials between 5.6 and $7.9 \mathrm{~m}$ in steps of $0.28 \mathrm{~m}$, and counterbalanced over conditions. The viewpoint moved through the environment at a constant speed of $1.12 \mathrm{~m} / \mathrm{sec}$. The object appeared on the poster $4 \mathrm{sec}$ before the onset of the turn.

No responses had to be made by the participants during the route learning session, and participants had no control over the timing of the video sequences to ensure the time spent at each trial type was matched. Additionally, the participants' viewing behavior was monitored during the entire scan period with an eye tracker (see Eye Tracking).

After the route learning session, a postlearning series of resting state scans were recorded using the same settings as in the prelearning session. After a 5-min break, participants performed a simple object recognition task (Figure 1B). Participants were instructed to indicate as 
quickly and accurately as possible whether they had seen an object in one of the mazes by pressing a yes or no response key. Responses were given with the index and middle finger of the right hand. A trial consisted of a fixation cross centered on the screen, followed by the presentation of an object for 500 msec shown from a canonical perspective on a white background. Thus, during scanning, no maze-related information was presented. The average ISI was $4000 \mathrm{msec}$, jittered between 3000 and $5000 \mathrm{msec}$ in steps of $250 \mathrm{msec}$, and counterbalanced over conditions. A total number of 252 stimuli were included in the recognition task, all 144 stimuli seen in the route learning session and 108 distractors. All stimuli were presented rapidly in a randomly intermixed order to prevent participants from anticipating and changing strategies for the different event types. Four sets of 36 stimuli from the following maze trial types were presented: D toy objects, D nontoy objects, ND toy objects, and ND nontoy objects. Three sets of 36 objects were included as distractor objects: novel toys, novel nontoys, and scrambled objects. The scrambled images were constructed from experimental objects using mosaic scrambling. Mean word frequency as well as frequency range was equal for all sets of objects.

Concluding the session in the scanner, an anatomical scan of each participant was obtained. After completing all fMRI scans, participants performed a source memory task outside the scanner. In this task, objects they had seen previously in the virtual museum were presented for 500 msec shown from a canonical perspective on a white background. Afterwards, participants made a button response, indicating in which of the four museum sections they had seen the object by pressing the button with the color corresponding to the maze (Figure 1B). The intertrial interval was $5000 \mathrm{msec}$, and objects were presented in a randomly intermixed order. All 144 stimuli presented during the route learning session were included in the maze source memory test. Four sets of 36 stimuli from one of the following maze trial types were presented: D toy objects, D nontoy objects, ND toy objects, and ND nontoy objects.

\section{Eye Tracking}

A commercial MRI compatible eye tracking device from SensoMotoric Instruments (S.M.I., Teltow, Germany; MEyeTrack-LR) mounted on the scanner bed was used to measure eye movements at a sampling rate of $50 \mathrm{~Hz}$. The participant's gaze location was recorded using infrared eye tracking during the entire route learning session to assess when and for how long each object was viewed. Simultaneous recording of onset and offset markers of videos enabled synchronization with stimulus presentation. Gaze fixation data were analyzed using in-house software implemented in Matlab 7.5 (The Mathworks, Inc., Natick, MA). The frames in which the objects became visible in the video sequences determined the onset timing of poster trials. For the analysis of poster viewing time, offsets of poster trials were defined as the moment the object on the poster was no longer visible. For each video frame within a trial in which the object was visible on the poster, the screen coordinates of the poster were extracted. A period of poster viewing was defined as the number of consecutive frames the gaze was on a poster coordinate. Poster views shorter than $60 \mathrm{msec}$ were discarded from the analysis. To precisely capture the moment participants process the object information, the actual trials entered into the fMRI analysis were defined as the onset of the first moment of poster viewing in each trial. Eye blinks and periods of data loss were identified by determining null data points and gaze locations far off the screen. Short blinks were removed from the signal using linear interpolation, whereas longer blinks and periods of data loss were excluded from the analyses. Data of two participants (both women) were not included in the analyses of the route learning session because of excessive eye tracker data loss.

\section{Image Acquisition}

All scans were obtained on a 3-T Trio MRI system with an eight-channel head array radio-frequency coil (Siemens, Erlangen, Germany). During the route learning session (lasting $32 \mathrm{~min}$ and $26 \mathrm{sec}$ ) and the object recognition test (lasting $19 \mathrm{~min}$ and $15 \mathrm{sec}$ ), a gradient-echo-planar scanning (EPI) sequence was used to acquire 36 axial slices per functional volume (voxel size $=3 \times 3 \times 3 \mathrm{~mm}$, repetition time $=2270 \mathrm{msec}$, field of view $=192 \mathrm{~mm}$, echo time $=30 \mathrm{msec}$, flip angle $=75^{\circ}$ ). In the two resting state sessions, each lasting $7 \mathrm{~min}$ and $25 \mathrm{sec}$, a twofold accelerated parallel imaging EPI sequence was used to acquire 265 functional whole-brain images $(39$ slices, voxel size $=$ $3.5 \times 3.5 \times 3.0 \mathrm{~mm}$, repetition time $=1680 \mathrm{msec}$, field of view $=224 \mathrm{~mm}$, echo time $=30 \mathrm{msec}$, flip angle $=70^{\circ}$ ). GRAPPA image reconstruction (Griswold et al., 2002) was used for accelerated scanning. After the acquisition of functional images, a high-resolution anatomical scan (T1weighted MP-RAGE, 192 slices) was acquired.

\section{Image Processing and Data Analysis}

Functional data were preprocessed and analyzed with SPM5 (www.fil.ion.ucl.ac.uk/spm). The first five volumes of each participant's EPI data were discarded to allow for T1 equilibration. The functional EPI-BOLD contrast images were realigned, and the mean of functional images was coregistered to the structural MRI using normalized mutual information optimization. Subsequently, functional images were slice-time corrected, spatially normalized, resampled to create 2-mm isotropic voxels, and transformed into a common stereotactic space, as defined by the SPM5 MNI T1 template, as well as spatially filtered by convolving the functional images with an isotropic 3-D Gaussian kernel (6 mm FWHM). 
Statistical analyses were performed in the context of the general linear model. For the analysis of the route learning session, we created regressors of interest based on the experimental conditions combined with the eye tracking data (see Eye Tracking). These conditions were based on the factors decision point (D and ND) and attention (toy and nontoy) and on empty trials. This resulted in the following conditions of interest: D toy objects, D nontoy objects, ND toy objects, ND nontoy objects, D empty trials, and ND empty trials. Regressors for empty decision points, that is, not containing a poster, and empty nondecision points were based on the average time of first poster viewing before the turn in the conditions containing posters. Additionally, regressors of no interest were included in the model to control for brain responses to certain events of no interest during the videos. These regressors included the following event types. Unviewed poster trials were modeled in a separate regressor with onsets based on the moment the object became visible on the poster. Regressors modeling the onset of the turn after the viewpoint had passed the poster were added separately for decision points and nondecision points. All regressors modeling events in the mazes were entered into the design matrix after each event-related stick function was convolved with a canonical hemodynamic response function. The six covariates corresponding to the movement parameters obtained from the realignment procedure were also included in the model. Statistical analysis in SPM5 included high-pass filtering (cutoff, $128 \mathrm{sec}$ ) to remove low-frequency confounds such as scanner drifts and correction for serial correlations using an autoregressive AR(1) model.

To analyze effects of subsequent memory (later remembered vs. later forgotten items) during the route learning session, a separate first-level statistical model was created in which all regressors modeling first views on the objects were divided according to whether the object on the poster was later remembered or forgotten. All other regressors included were the same as in the other model.

For the analysis of the object recognition session, objects from the different stimulus sets were modeled into four conditions of previously seen objects and three distractors: novel toys, novel nontoys, and scrambled objects. To examine the effects of successful memory retrieval, a separate first-level model was created in which each regressor for the objects previously seen in the museum was divided according to whether the object was remembered or forgotten. To test for memory effects during encoding and retrieval in the PHG, ROI analyses were performed on the activated regions in this area for the contrast $\mathrm{D}$ points $>$ ND points during the recognition session.

Data from the resting state session were preprocessed in the same way as described above for the task session, except that a different isotropic 3-D Gaussian kernel was used ( $8 \mathrm{~mm}$ FWHM). Also, the images were low-pass filtered using a fifth-order Butterworth filter to retain frequencies below $0.1 \mathrm{~Hz}$, because the correlations between intrinsic fluctuations are specific to this frequency range (Fox \& Raichle, 2007; Biswal et al., 1995). The resting state sessions were modeled into a single model to compare differences in functional connectivity postroute compared with preroute learning. A seed region of the PHG was created by overlapping the anteriorly activated regions from the $\mathrm{D}$ objects > ND objects contrast in the second-level analyses of the route learning and object recognition sessions. For each participant, a first-level model was created in which the filtered mean time courses of the overlapping region between the participant-specific segmented gray matter maps and the PHG seed region were entered separately for both sessions. Regressors of no interest were included to control for global signal effects, containing the average BOLD signal for all gray matter voxels for every volume, the average signal for all white matter voxels, the average signal for all cerebrospinal fluid voxels, and the average signal for a blank portion of the MRIs (Out of Brain signal). Furthermore, head motion parameters for both sessions were added to the model.

The single-subject parameter estimates for the conditions of interest from each session were included in subsequent random effects analyses. For these second-level analyses, factorial ANOVAs were used. In the analysis of the route learning session, decision point (D objects and ND objects) and poster type (toy poster, nontoy poster, and empty) were entered as within-subject factors. The second-level analysis of the object recognition session contained decision point (D objects and ND objects) and toy (toy and nontoy poster) as within-subject factors. For the resting state sessions, the prelearning and postlearning parameter estimates, expressing connectivity in each voxel with the parahippocampal seed region within that session, were entered in a second-level factorial model containing the factor session (prelearning and postlearning). Covariates of interest were added to the model, modeling subject performance on the recognition test and the maze source memory test, and their SBSOD scores. The mean SBSOD score was $67.5 \pm 13.77$ (mean male score $=72.5$, mean female score $=62.5, t(22)=-1.873, p=.074)$. All secondlevel group analyses were performed at the whole-brain level with a significance threshold at the cluster level of $p<.05$ family-wise error rate corrected at the whole-brain threshold of $p<.001$ uncorrected (Hayasaka \& Nichols, 2003). To look at the effects of memory during the object recognition session and the $\mathrm{D}$ point $>$ ND point objects during the route learning session, we performed ROI analyses in the brain regions activated for the $\mathrm{D}$ point $>\mathrm{ND}$ point objects during the object recognition session. Furthermore, based on our a priori hypothesis about the relationship between navigational ability and involvement of the hippocampus, we also performed ROI analysis on a hippocampal region derived from the literature (Iaria et al., 2003) for this contrast. For all ROI analyses, we report clusters significant at $p<.001$ uncorrected for multiple comparisons that survive small volume correction (SVC) for multiple comparisons, which corrects for a reduced search 
region based on the size of the region under investigation. Mean parameter estimates, used for illustrative purposes, were extracted using MarsBaR (Brett, Anton, Valabregue, \& Poline, 2002). Visualizations of activations were created using MRIcron (www.cabiatl.com/mricro/mricron/) by superimposing statistical parametric maps thresholded at $p<$ .001, uncorrected, onto a canonical T1-weighted image in standard MNI152 space.

\section{RESULTS}

\section{Behavioral Results}

\section{Eye Tracking}

The average time spent viewing each poster was $1769 \mathrm{msec}$ (Figure 2). The viewing time data were entered into an ANOVA with the factors decision point (D objects and ND objects) and attention (toy objects and nontoys objects). The data showed a main effect of attention during learning $(F(1,21)=20.20, p<.001)$. This main effect showed that participants looked longer at toys than at nontoys $(p<$ $.01)$. No main effect of decision point was observed $(F(1$, $21)=2.22, n s)$. An interaction was observed between decision point and attention $(F(1,21)=13.53, p<.01)$. Toys at nondecision points $(M=1968 \mathrm{msec}, S D=119 \mathrm{msec})$ were viewed longer than toys at decision points $(M=$ $1862 \mathrm{msec}, S D=121 \mathrm{msec} ; t(21)=3.55, p<.01$ ). Toys at decision points were viewed longer than nontoys at nondecision points $(M=1723 \mathrm{msec}, S D=107 \mathrm{msec}$; $t(21)=$ $3.04, p<.01)$. Toys at nondecision points were viewed longer than nontoys at decision points $(M=1772 \mathrm{msec}$, $S D=100$ msec; $t(21)=5.17, p<.001)$. Finally, toys at nondecision points were viewed longer than nontoys at nondecision points $(t(21)=7.55, p<.001)$.

\section{Recognition Test in the Scanner}

Task performance during the recognition task in the scanner was above chance (77\% correct, see Table 1). Although

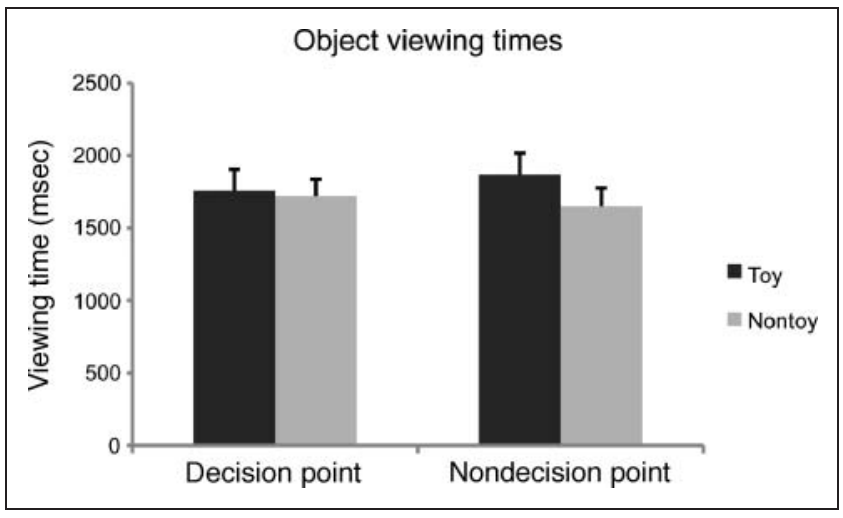

Figure 2. Viewing behavior. Average viewing times for objects on posters. Bars indicate standard error across participants.
Table 1. Recognition Performance during the Object Recognition Session

\begin{tabular}{ccccc}
\hline \multicolumn{2}{c}{ Objects from Mazes } & & \multicolumn{2}{c}{ Distractor Objects } \\
\cline { 1 - 1 } Hits (\%) & Misses (\%) & & Correct Rejections (\%) & False Alarms (\%) \\
\hline $65 \pm 10$ & $35 \pm 10$ & $90 \pm 7$ & $10 \pm 7$ \\
\hline
\end{tabular}

The table shows means and standard deviations of recognition performance.

participants had a considerable false alarm rate, average recognition performance as defined by $d^{\prime}$ was $1.68 \pm$ 0.52 . An ANOVA on the $d^{\prime}$ values showed a main effect of Attention during Learning $(F(1,23)=7.86, p<.02)$. Performance was higher for toys than for nontoys (mean $d^{\prime}$ values were 1.99 and $\left.1.64, t(23)=2.78, p<.02\right)$. No significant main effect of Decision Point and no significant interaction were observed.

Response times for correct answers showed an effect of Attention during Study $(F(1,23)=15.03, p<.001)$. Response times were faster for toys than for nontoys (mean response latencies were 945 and $967 \mathrm{msec}, t(23)=-2.284$, $p<.05)$. No significant main effect of Decision Point and no significant interaction were observed.

\section{Maze Source Memory Test outside the Scanner}

Task performance during the maze source memory test outside the scanner was above chance (31\% correct \pm $4 \% ; t(23)=7.56, p<.001$; chance level $=25 \%$ ). An ANOVA on the accuracies with the factors Decision Point and Attention showed no main effects and no interactions. Response times showed no main effects of Decision Point and Attention and no interaction.

\section{fMRI Results}

\section{Object Recognition Session}

To investigate which brain regions selectively respond to the navigational relevance of objects during recognition, we compared fMRI to D objects with responses to ND objects. This main effect revealed higher activations in the bilateral PHG and bilateral middle occipital gyrus (Table 2A). We compared toy objects with nontoy objects to investigate the effects of attention during recognition in a wholebrain analysis. Significant increases were found in bilateral fusiform gyrus, the right cuneus, and right middle temporal gyrus (see Table 2A). Negative effects of attention were found in the left fusiform gyrus.

To investigate whether the effects of navigational relevance in the PHG depend on the ability to recall having seen objects in the museum, we modeled remembered and forgotten items within each condition in a separate statistical model (see Methods). Comparing remembered D objects with ND objects revealed a significant effect in the right PHG, whereas a comparison between forgotten 
Table 2A. Brain Regions Showing Significant Activations during Object Recognition

\begin{tabular}{|c|c|c|c|c|c|c|}
\hline Contrast & Region & $k$ & $x$ & $y$ & $z$ & Peak t Score \\
\hline \multirow[t]{4}{*}{$\mathrm{D}$ point $>$ ND point objects } & Left middle and superior occipital gyrus & $485^{* * *}$ & -26 & -92 & 18 & 5.73 \\
\hline & Right middle occipital gyrus & $505 * * *$ & 30 & -86 & 30 & 5.44 \\
\hline & Left parahippocampal gyrus & $137 * *$ & -32 & -42 & -8 & 4.63 \\
\hline & Right parahippocampal gyrus & $155^{* *}$ & 32 & -44 & -6 & 4.34 \\
\hline \multirow[t]{4}{*}{ Toys $>$ Nontoys } & Right fusiform gyrus & $412 * * *$ & 40 & -52 & -16 & 7.91 \\
\hline & Right middle temporal gyrus & $527 * * *$ & 52 & -72 & 6 & 6.78 \\
\hline & Left fusiform gyrus & $164 * *$ & -36 & -46 & -22 & 6.13 \\
\hline & Right superior occipital gyrus/right cuneus & $291 * * *$ & 20 & -94 & 16 & 4.76 \\
\hline Nontoys $>$ Toys & Left lingual gyrus/left fusiform gyrus & $98 *$ & -30 & -48 & -2 & 5.00 \\
\hline
\end{tabular}

D objects with ND objects revealed a significant effect in the left PHG (Table 2B).

\section{Route Learning Session}

To reveal the brain regions that respond to the navigational relevance of objects during the first encounter, D objects were contrasted with ND objects from trials in which a poster was presented. This comparison revealed an increase in activity in the bilateral middle and superior occipital gyrus, bilateral PHG, bilateral lingual gyrus, right precuneus, left middle frontal gyrus, and left cuneus (Table 3A and Figure 3). This comparison, however, possibly confounds the encoding of objects with the encoding of scenes. Decision points are usually visually more complex than nondecision points, which might account for the higher activations found in the previous contrast. Indeed, we also found higher activations for empty decision points compared with empty nondecision points in many of the same regions activated in the D versus ND poster contrast: the bilateral middle occipital gyrus, bilateral middle frontal gyrus, left superior parietal lobule, right superior occipital gyrus, right fusiform gyrus, right precuneus and right PHG (Table 3A). A contrast between posters and empty trials revealed increased activity in bilateral fusiform gyrus, left middle occipital gyrus, left inferior frontal gyrus, left insula, left SMA, left cuneus, right calcarine gyrus, and right angular gyrus (Table 3A). These activations included the bilateral PHG, as shown by an analysis within the activated region in the bilateral PHG from the D points $>$ ND points contrast during the recognition task (Table $3 \mathrm{~A}$ ).

Comparing fMRI responses to toys with those to nontoys revealed increased activity in the left medial frontal gyrus, right inferior and middle occipital gyrus, left superior parietal lobule, left insula, left inferior frontal gyrus, right angular gyrus, left middle occipital gyrus and left inferior occipital gyrus (Table 3A). Importantly, an analysis within the bilateral PHG region sensitive to the navigational relevance of objects during recognition revealed no clusters within this reduced search region. No significant regions showing higher activations for nontoys compared with toys were observed.

Table 2B. Significant Object Recognition Results within Bilateral Parahippocampal Gyrus, Events Separated According to Successful Memory Retrieval

\begin{tabular}{|c|c|c|c|c|c|c|}
\hline Contrast & Region & $k$ & $x$ & $y$ & $z$ & Peak t Score \\
\hline Remembered D objects $>$ Remembered ND objects & Right Parahippocampal Gyrus & $5^{*}$ & 24 & -36 & -14 & 3.52 \\
\hline \multirow[t]{2}{*}{ Forgotten D objects $>$ Forgotten ND objects } & Left Parahippocampal Gyrus & $23 * *$ & -34 & -44 & -6 & 4.25 \\
\hline & Left Parahippocampal Gyrus & $15^{*}$ & -28 & -58 & -6 & 3.82 \\
\hline
\end{tabular}

The $x, y, z$ coordinates of the local maxima are given in MNI standard space coordinates. Whole brain threshold $p<.001$ uncorrected. SVC on the bilateral PHG region activated in the D objects $>$ ND objects contrast from the retrieval session. $k=$ cluster size.

$* p<.05$ small volume corrected.

$* * p<.01$ small volume corrected. 
Table 3A. Brain Regions Showing Significant Activations during the Route Learning Session

\begin{tabular}{|c|c|c|c|c|c|c|}
\hline Contrast & Region & $k$ & $x$ & $y$ & $z$ & Peak t Score \\
\hline \multirow[t]{10}{*}{ D points $>$ ND points objects } & Left middle/superior occipital gyrus & $1545 * * * * *$ & -22 & -94 & 26 & 8.05 \\
\hline & Right middle/superior occipital gyrus & $1723 * * * * *$ & 28 & -92 & 26 & 6.91 \\
\hline & Right lingual gyrus & $871 * * * * *$ & 16 & -72 & -10 & 6.15 \\
\hline & Right parahippocampal gyrus & & 28 & -42 & -6 & 5.50 \\
\hline & Left lingual gyrus & $286 * * * * *$ & -28 & -46 & -6 & 5.98 \\
\hline & Left parahippocampal gyrus & & -20 & -38 & -10 & 3.64 \\
\hline & Right precuneus & $260 * * * * *$ & 20 & -56 & 22 & 5.71 \\
\hline & Left cuneus/calcarine sulcus & $173 * * *$ & -18 & -66 & 22 & 4.92 \\
\hline & Left middle frontal gyrus & $180 * * *$ & -26 & -2 & 56 & 4.77 \\
\hline & Left lingual gyrus & $137^{*}$ & -14 & -76 & -14 & 4.28 \\
\hline \multirow[t]{9}{*}{ D empty $>$ ND empty } & Left middle occipital gyrus & $1364 * * * * *$ & -28 & -86 & 26 & 6.81 \\
\hline & Left superior parietal lobule & & -22 & -78 & 46 & 6.59 \\
\hline & Right middle occipital gyrus & $1913 * * * * *$ & 32 & -80 & 30 & 6.75 \\
\hline & Right superior occipital gyrus & & 28 & -88 & 32 & 6.55 \\
\hline & Left middle frontal gyrus & $350 * * * * *$ & -28 & 0 & 56 & 6.07 \\
\hline & Right precuneus & $168 * * *$ & 18 & -58 & 22 & 5.39 \\
\hline & Right parahippocampal gyrus & $178 * * *$ & 28 & -42 & -8 & 4.86 \\
\hline & Right fusiform gyrus & & 30 & -52 & -6 & 4.29 \\
\hline & Right superior frontal gyrus & $137^{*}$ & 24 & 2 & 52 & 4.59 \\
\hline \multirow[t]{4}{*}{ (SVC on bil. PHG from recognition) } & Right parahippocampal gyrus & $63 * * * *$ & 28 & -44 & -8 & 4.67 \\
\hline & Left parahippocampal gyrus & $36 * * * *$ & -26 & -44 & -6 & 4.24 \\
\hline & Right parahippocampal gyrus & $1 * *$ & 22 & -40 & -8 & 3.46 \\
\hline & Right parahippocampal gyrus & $1 * *$ & 24 & -36 & -12 & 3.20 \\
\hline \multirow[t]{9}{*}{ Posters > empty } & Left fusiform gyrus & $3688 * * * * *$ & -30 & -38 & -22 & 7.35 \\
\hline & Middle occipital gyrus & & -46 & -66 & -12 & 6.69 \\
\hline & Right fusiform gyrus & $3478 * * * * *$ & 44 & -60 & -14 & 6.94 \\
\hline & Left interior frontal gyrus & $312 * * * * *$ & -42 & 8 & 30 & 4.34 \\
\hline & Left SMA & $306 * * * * *$ & 0 & 10 & 54 & 4.33 \\
\hline & Left cuneus & $229 * * *$ & -2 & -78 & 16 & 4.17 \\
\hline & Right calcarine gyrus & $159 * * *$ & 22 & -66 & 8 & 4.06 \\
\hline & Right angular gyrus & $116^{*}$ & 32 & -58 & 50 & 4.03 \\
\hline & Left insula & $117^{*}$ & -32 & 24 & 4 & 3.92 \\
\hline \multirow[t]{3}{*}{ (SVC on bil. PHG from recognition) } & Right parahippocampal gyrus & $24 * * * *$ & 26 & -32 & -20 & 5.80 \\
\hline & Left parahippocampal gyrus & $4 * *$ & -28 & -60 & -12 & 3.68 \\
\hline & Left parahippocampal gyrus & $1 * *$ & 32 & -38 & -14 & 3.26 \\
\hline \multirow[t]{4}{*}{ Toys $>$ nontoys } & Left medial frontal gyrus/SMA & $274 * * * * *$ & -8 & 26 & 40 & 5.15 \\
\hline & Right inferior occipital gyrus & $1738 * * * * *$ & 50 & -64 & -16 & 4.89 \\
\hline & Right middle occipital gyrus & & 50 & -76 & 6 & 4.74 \\
\hline & Left superior parietal lobule & $267 * * * * *$ & -30 & -64 & 50 & 4.83 \\
\hline
\end{tabular}


Table 3A. (continued)

\begin{tabular}{|c|c|c|c|c|c|c|}
\hline Contrast & Region & $k$ & $x$ & $y$ & $z$ & Peak t Score \\
\hline & Left insula & $127^{*}$ & -34 & 18 & -4 & 4.73 \\
\hline & Left precentral gyrus/inferior frontal gyrus & $682 * * * * *$ & -38 & 4 & 36 & 4.51 \\
\hline & Right angular gyrus & $216 * * *$ & 32 & -62 & 42 & 4.12 \\
\hline & Left middle occipital gyrus & $132 *$ & -30 & -96 & -4 & 4.01 \\
\hline & Left inferior occipital gyrus & $103 *$ & -38 & -86 & -14 & 3.80 \\
\hline
\end{tabular}

The $x, y, z$ coordinates of the local maxima are given in MNI standard space coordinates. Whole brain threshold $p<.001$, uncorrected. SVC analyses were carried out on the bilateral PHG region activated in the D objects $>$ ND objects contrast from the retrieval session. $k=$ cluster size.

$* p<.05$ at the cluster level.

$* * p<.05$ small volume corrected.

$* * * p<.01$ at the cluster level.

$* * * * p<.01$ small volume corrected

$* * * * * p<.001$ at the cluster level.

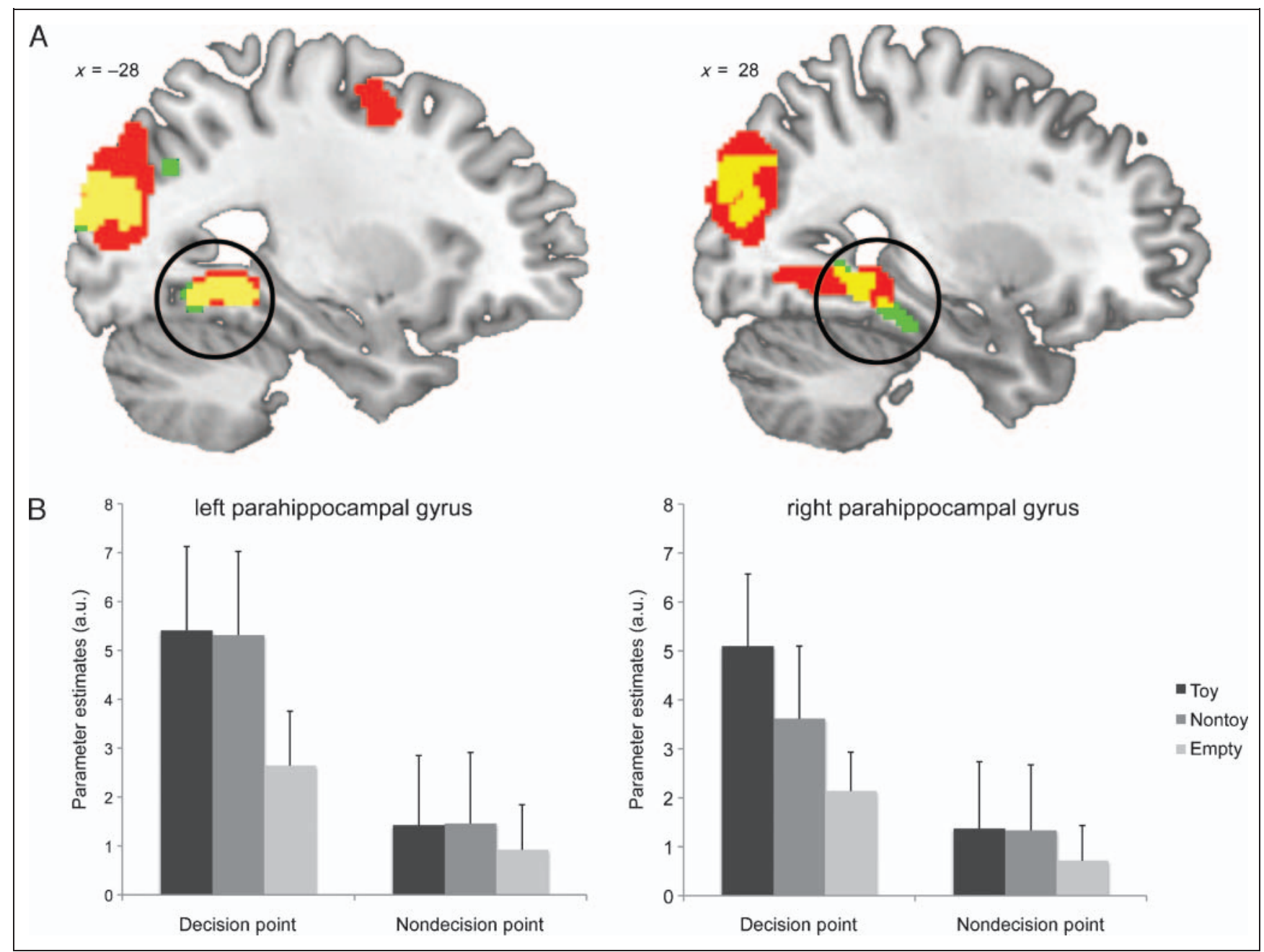

Figure 3. Brain activations during route learning and recognition sessions. (A) Bilateral PHG showing an increased response for decision point as compared with nondecision point objects. Significantly activated regions shown during the route learning session (in red), during the object recognition session (in green), and during both (in yellow). All statistical parametric maps are thresholded at $p<.001$, uncorrected at the voxel level, with a cluster size that exceeds 100 voxels, showing the significant clusters. For formal statistical tests, see Tables 2 and 3. (B) Regionally averaged parameter estimates during the route learning session for the left and right PHG for attended (toys), nonattended (nontoys), and empty trials. Parameter estimates were extracted from the PHG regions that showed a stronger activation for decision point as compared with nondecision point objects during the object recognition session. 
We investigated effects of subsequent memory in an ANOVA containing the factors decision point (D objects vs. ND objects), attention (toy vs. nontoy objects) and subsequent memory (later remembered vs. later forgotten objects). Because of our interest in the PHG, we restricted our analysis to the PHG regions showing sensitivity for the navigational relevance during recognition (Figure 3). Increased activity for D objects compared with ND objects was observed for both later remembered and later forgotten objects (Table 3B). Furthermore, a main effect of later remembered versus later forgotten objects was observed in the right PHG (Table 3B).

\section{Resting State}

To investigate the effect of spatial learning on the functional connectivity with the PHG, we created a bilateral seed region by taking the overlapping PHG voxels from the route learning and recognition sessions that showed sensitivity to the navigational relevance of objects during both the recognition and the route learning sessions (Figure 3; yellow regions inside black circles). To investigate whether functional connectivity changes between the PHG and other brain regions predicted subsequent task performance or correlated with navigational ability, we created a model containing prelearning and postlearning PHG time courses. Additionally, we added the following covariates: subsequent performance during the recognition test, subsequent performance on the maze source memory test, and the score of each participant on the SBSOD questionnaire (see Methods). No clusters were found that showed a significantly stronger connectivity to the PHG postlearning compared with prelearning learning or vice versa. We further tested whether spatial ability correlated with learning-induced connectivity. Specifically, we looked at the hippocampus, which is associated with the use of a sur- vey (allocentric, world-centered) navigational strategy (Iaria et al., 2003). On the basis of the results of this study, we chose an ROI, centered on the peak activity in the right hippocampus in the survey strategy group during the first experimental trial compared with control trials. A spherical ROI (radius $=10 \mathrm{~mm}$ ) was centered at the local maximum $[32,-14,-20]$ of this contrast from a study by Iaria et al. (2003). Indeed, we found a stronger positive correlation between postlearning compared with prelearning connectivity and the navigational ability measured by the SBSOD scale $\left(P_{\text {SVC }}=.031\right.$, peak $t$ value $=3.92$ at location $[34,-6$, $-14]$, nine voxels). Of these nine voxels, five voxels overlapped with the anatomical mask of the right hippocampus in the AAL atlas (Tzourio-Mazoyer et al., 2002). Furthermore, we were interested whether regions displayed a negative correlation between learning-induced connectivity changes and their navigational ability. Here, we found a negative correlation (cluster $p=.013$, whole-brain corrected for multiple comparisons, peak $t$ value $=6.66$ at location $[18,20,14]$, 183 voxels) between the right caudate nucleus and the PHG postlearning minus prelearning connectivity and the navigational ability scores of subjects (Figure 4). No other significant regions were found in this contrast. Also, no regions showed significant learning-induced connectivity changes to the PHG that correlated positively or negatively with one of the memory performance scores.

To exclude the possibility that inclusion of our task performance measures as covariates absorbed shared covariance and therefore obscured potentially interesting results, we also analyzed the data with only the navigational ability score as a covariate. The correlation results between the connectivity and the SBSOD navigational ability remained: a negative correlation between SBSOD and connectivity with the PHG was observed in the right caudate nucleus (cluster $p=.026$, whole-brain corrected, peak $t$ value $=$ 6.23 at location $[16,20,14])$ and a positive correlation

Table 3B. Significant Route Learning Results within Bilateral Parahippocampal Gyrus, Events Separated according to Subsequent Memory

\begin{tabular}{|c|c|c|c|c|c|c|}
\hline Contrast & Region & $k$ & $x$ & $y$ & $z$ & Peak t Score \\
\hline \multirow[t]{2}{*}{ D remembered $>$ ND remembered } & Left parahippocampal gyrus & $54 * *$ & -28 & -46 & -6 & 4.61 \\
\hline & Right parahippocampal gyrus & $45^{* *}$ & 26 & -42 & -6 & 4.10 \\
\hline \multirow[t]{4}{*}{ D forgotten $>$ ND forgotten } & Right parahippocampal gyrus & $60 * *$ & 32 & -40 & -6 & 4.59 \\
\hline & Left parahippocampal gyrus & $77 * *$ & -30 & -44 & -4 & 4.40 \\
\hline & Right parahippocampal gyrus & $1 *$ & 22 & -40 & -8 & 3.68 \\
\hline & Right fusiform gyrus & $7^{*}$ & 26 & -38 & -12 & 3.64 \\
\hline \multirow[t]{2}{*}{ Later remembered objects > Later forgotten objects } & Right parahippocampal gyrus & $2 *$ & 26 & -30 & -22 & 3.47 \\
\hline & Right parahippocampal gyrus & $1 *$ & 32 & -38 & -14 & 3.35 \\
\hline
\end{tabular}

The peak $x, y, z$ coordinates are given in MNI standard space coordinates. Whole-brain threshold $p<.001$, uncorrected. SVC on the bilateral PHG region activated in the D objects $>$ ND objects contrast from the retrieval session. $k=$ cluster size.

$* p<.05$ small volume corrected.

$* * p<.01$ small volume corrected. 


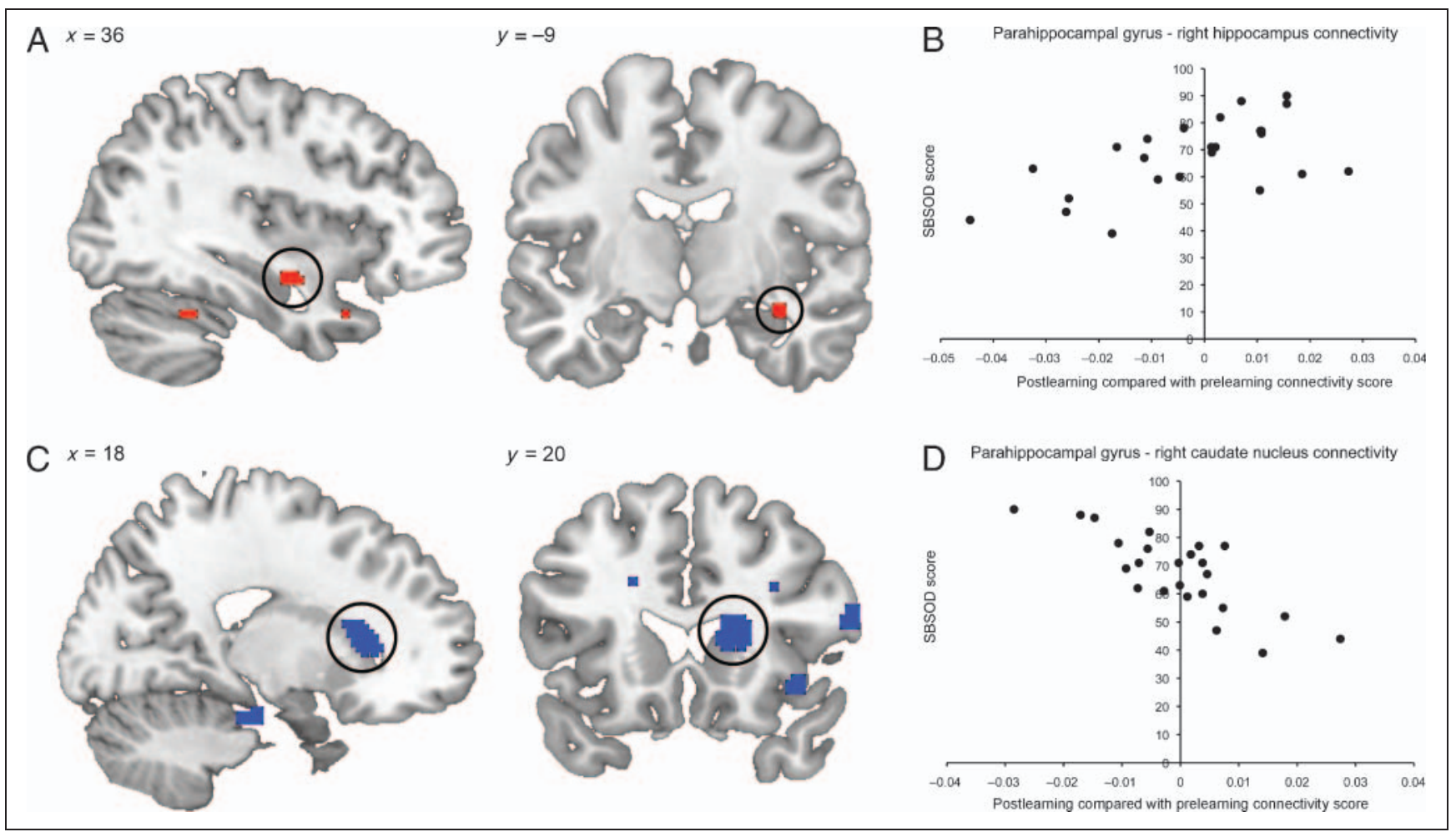

Figure 4. Resting state connectivity with bilateral PHG postlearning compared with prelearning and regions correlated with navigation ability. (A) A positive correlation between postlearning compared with prelearning connectivity to PHG was found in right hippocampus. (B) SBSOD (self-reported navigational ability, see Methods) score as a function of regionally averaged right hippocampus postlearning compared with prelearning connectivity with PHG. (C) A negative correlation between postlearning compared with prelearning connectivity to PHG was found in right caudate nucleus. (D) SBSOD score as a function of regionally averaged right caudate nucleus postlearning compared with prelearning connectivity with PHG. Statistical maps thresholded at $p<.001$, uncorrected.

between SBSOD and connectivity with the PHG was still observed in our hippocampus ROI $\left(P_{\mathrm{SVC}}=.029\right.$, peak $t$ value $=3.74$ at location $[34,-8,-14])$. When comparing overall connectivity with the PHG prelearning greater than postlearning, we found a cerebellum region (cluster $p=.032$, peak $t$ value $=4.81$ at location $[28,-54,-46]$ ) . Connectivity with the PHG postlearning was significantly increased compared with prelearning in a region overlapping the thalamus bilaterally (cluster $p=.013$, peak $t$ value $=$ 4.37 at location $[6,-12,20])$. Additionally, we found a significant cluster in the cerebellum (cluster $p=.012$, whole-brain corrected, peak $t$ value $=5.04$ at location $[28,-52,-50]$ )

\section{DISCUSSION}

In the present event-related fMRI study, we investigated how the selective neural representation of objects relevant for navigation is established and how learning influences resting state functional connectivity. Participants watched a route through a virtual environment inside the MRI scanner. They were instructed to remember the route and all objects appearing on posters along it. To compare general and memory-related differences in functional connectivity as a result of spatial learning, we recorded resting state scans before and after route learning. Afterwards, participants performed an object recognition task in the scanner.
Outside the scanner, they performed a source memory test on the previously learned objects and answered questions about their navigational skills.

In line with previous studies (Janzen \& Jansen, 2010; Schinazi \& Epstein, 2010; Janzen et al., 2007, 2008; Janzen \& Weststeijn, 2007; Janzen \& van Turennout, 2004), results from the object recognition session show involvement of the bilateral PHG for decision point objects as compared with nondecision point objects.

During learning, the PHG showed increased activity for objects at decision points at the first moment the objects were encountered. This difference could not be explained by differences in viewing behavior and was independent of paying attention to an object. The PHG is activated by viewing scenes and has been shown to be involved in the first-person encoding of novel environments with objects that could be used to guide navigation (Epstein, 2008; Shelton \& Gabrieli, 2002; Epstein \& Kanwisher, 1998; Maguire et al., 1998; Aguirre, Detre, Alsop, \& D'Esposito, 1996). However, these studies did not allow distinguishing between the roles of environmental features and that of objects, because participants could have used both these sources of information to find their way. Recent studies, however, have tried to disentangle these possibilities by suggesting the PHG involvement in the encoding of viewercentered geometrical spatial information in an environment 
without objects (Weniger et al., 2010) and in encoding based on object locations in an open field environment (Baumann et al., 2010). Compared with the environments used in these studies, the present study used a complex environment containing numerous objects that differed in their usefulness for guiding navigation. Our present findings, therefore, extend the previous results by showing that the PHG distinguishes between the navigational relevance of objects during object-based encoding.

Looking at locations containing objects versus looking at locations containing no objects also resulted in higher PHG activity. Previous studies found no difference in PHG responses between empty scenes and scenes with objects in them (Epstein, Harris, Stanley, \& Kanwisher, 1999; Epstein \& Kanwisher, 1998). Participants in our study had to remember all objects they encountered along the route, suggesting the PHG is involved in encoding objects in their context. We additionally show that the PHG was activated more strongly by empty decision points compared with empty nondecision points. This effect shows that a decision point per se activates the PHG during route learning. Whether the navigational relevance of a decision point by itself or additional factors, for example, differences in visual features between decision points and nondecision points (Chai, Ofen, Jacobs, \& Gabrieli, 2010) are represented in this brain region needs to be investigated in future studies.

Activity in the PHG during route learning predicted the chance of successfully remembering an object during the object recognition session. Other examples of such a subsequent memory effect in the PHG have been found in fMRI studies where participants had to encode pictures of landscapes (for a review, see Davachi, 2006). In line with the present findings, Baumann et al. (2010) report that elevated PHG responses during route learning predict successful memory-guided navigation based on landmarks in a simple open-field environment.

A decision point effect was observed during route learning for both later remembered and later forgotten items. This extends the results obtained in previous studies (Janzen et al., 2007; Janzen \& van Turennout, 2004), where it was shown that the decision point effect during recognition is independent of conscious recollection of the objects. In the object recognition session, we only observed increased activation in the right PHG for remembered decision point objects versus nondecision point objects and increased activation only in the left PHG for forgotten decision point objects compared with nondecision point objects. Previous results from our group have shown bilateral PHG activation for remembered and forgotten items (Janzen et al., 2007) as well as unilateral PHG activation (Janzen \& van Turennout, 2004). Because the trials within conditions were split up according to whether the items were remembered or forgotten, we think the unilateral observation of these effects is a power issue rather than a qualitative difference in PHG functioning.

The effects of route learning on functional brain connectivity during rest were examined by comparing resting state scans acquired directly prelearning and postlearning. The part of PHG that was involved in both encoding and retrieving navigationally relevant information was used as a seed region to determine functional connectivity changes induced by spatial learning. No brain regions were found that showed a general increase in connectivity with the PHG postlearning compared with prelearning. We did, however, observe postlearning compared with prelearning connectivity changes that correlated with participants' selfreported navigational abilities. The SBSOD score correlates with tests of acquired environmental knowledge on different scales in real as well as in virtual environments (Hegarty, Montello, Richardson, Ishikawa, \& Lovelace, 2006; Hegarty et al., 2002), indicating that people have a good subjective awareness of their spatial abilities. Furthermore, someone's sense of direction correlates positively with the use of a survey navigational strategy (Prestopnik \& RoskosEwoldsen, 2000) and a poor sense of direction group makes less use of a survey strategy compared with a group with a good sense of direction (Kato \& Takeuchi, 2003). Additional support for a relation between a preferred survey strategy and navigational ability comes from a study showing that spontaneous adopters of a survey strategy in a small maze outperformed spontaneous route strategy (egocentric, response-based) users in a different, large-scale virtual environment (Etchamendy \& Bohbot, 2007). Therefore, although we cannot relate our connectivity results with a direct measure of map formation, good navigators are more likely to build survey representations of their environment.

Given that the neural correlates of using a survey strategy have been found in the hippocampus in both humans (Doeller et al., 2008; Hartley et al., 2003; Iaria et al., 2003) and rodents (Packard \& McGaugh, 1996; McDonald \& White, 1994; O'Keefe \& Nadel, 1978), we performed an ROI analysis in that region. We found a positive correlation between the postlearning compared with prelearning connectivity with the PHG and the navigational ability of participants. The activated region overlapping with the hippocampus is not near other gray matter regions and is unlikely to arise from surrounding white matter. We, therefore, believe that the neural origin of the observed cluster is in the hippocampus. Navigational ability and these connectivity changes were negatively correlated with the caudate nucleus. These findings are in line with a distinction between the hippocampus and the caudate nucleus supporting survey and route navigational strategies, respectively (Hartley et al., 2003; Iaria et al., 2003; Packard \& McGaugh, 1996; O'Keefe \& Nadel, 1978). This distinction is also supported by structural differences in gray matter volume in the hippocampus and the caudate nucleus, which correlate with the use of survey and route strategies (Bohbot, Lerch, Thorndycraft, Iaria, \& Zijdenbos, 2007). Both hippocampal and striatal spatial learning systems seem to work in parallel (Voermans et al., 2004), having an influence on behavior that is proportional with their activation (Doeller et al., 2008). The amount to which the PHG communicates with these regions after the learning of 
object-related spatial information could thus determine one's preferred navigation strategy.

Several studies suggest a functional distinction between the anterior and posterior hippocampus with regard to navigation. Involvement of the anterior hippocampus, which is also investigated in this study, is more pronounced during map formation (Wolbers \& Büchel, 2005), whereas the posterior hippocampus is involved when using it (Iaria, Chen, Guariglia, Ptito, \& Petrides, 2007).

There are several studies reporting a relationship between self-reported navigational abilities and spatial representations in the brain. Janzen et al. (2008) found that a consolidation effect in the hippocampus (activation for remote objects compared with recent objects) for objects previously encountered in a virtual environment correlated with self-reported navigational ability. This study also shows that the decision point effect in the PHG increases over time for good navigators. Similarly, Epstein, Higgins, and Thompson-Schill (2005) found better PHG representations for places and views in (self-reported) good navigators compared with bad navigators. Several studies also found that good navigators seem to be able to flexibly shift between both map-based and response-based strategies, depending on whichever is the most appropriate in a given situation (Etchamendy \& Bohbot, 2007; Hartley et al., 2003). Our results show differences in off-line reprocessing related to a person's navigational abilities directly following a spatial learning experience, suggesting that good navigators encode spatial representations more efficiently and integrate them over time into hippocampal representations containing both objects and maps. Bad navigators lack this flexibility, which might be because of a stronger crosstalk between the PHG and regions that subserve response-based navigation such as the caudate.

In summary, these results indicate that the PHG is involved in the processing of information relevant for navigation during retrieval of this information and on the first moment this information is encountered. These findings also show that connectivity changes after spatial learning between the PHG and the caudate nucleus and the hippocampus are related to a person's navigational ability. This spatial information flow in the resting brain as a result of learning varies as a function of navigational ability and provides valuable insights into the neural correlates of individual differences in spatial ability.

\section{Acknowledgments}

We thank Janneke van Ekert and Atsuko Takashima for helpful comments on this manuscript. This work was supported by the Netherlands Organization for Scientific Research (Vidi Grant No. 452-07-015) and the European Commission (ERC Starting Independent Researcher Grant No. 204643).

Reprint requests should be sent to Joost Wegman, Centre for Cognitive Neuroimaging, Donders Institute for Brain, Cognition and Behaviour, Radboud University Nijmegen, P.O. Box 9101, 6500 HB Nijmegen, The Netherlands, or via e-mail: joost.wegman@ donders.ru.nl.

\section{REFERENCES}

Aguirre, G. K., Detre, J. A., Alsop, D. C., \& D’Esposito, M. (1996). The parahippocampus subserves topographical learning in man. Cerebral Cortex, 6, 823-829.

Albert, N. B., Robertson, E. M., \& Miall, R. C. (2009). The resting human brain and motor learning. Current Biology, 19, 1023-1027.

Baumann, O., Chan, E., \& Mattingley, J. B. (2010). Dissociable neural circuits for encoding and retrieval of object locations during active navigation in humans. Neuroimage, 49, 2816-2825.

Biswal, B., Yetkin, F. Z., Haughton, V. M., \& Hyde, J. S. (1995). Functional connectivity in the motor cortex of resting human brain using echo-planar MRI. Magnetic Resonance in Medicine, 34, 537-541.

Bohbot, V. D., Lerch, J., Thorndycraft, B., Iaria, G., \& Zijdenbos, A. P. (2007). Gray matter differences correlate with spontaneous strategies in a human virtual navigation task. Journal of Neuroscience, 27, 10078-10083.

Brett, M., Anton, J.-L., Valabregue, R., \& Poline, J.-B. (2002). Region of interest analysis using an SPM toolbox [Abstract]. Paper presented at the International Conference on Functional Mapping of the Human Brain, Sendai, Japan.

Chai, X. J., Ofen, N., Jacobs, L. F., \& Gabrieli, J. D. E. (2010). Scene complexity: Influence on perception, memory, and development in the medial temporal lobe. Frontiers in Human Neuroscience, 4, 21.

Davachi, L. (2006). Item, context and relational episodic encoding in humans. Current Opinion in Neurobiology, 16, 693-700.

Doeller, C., King, J., \& Burgess, N. (2008). Parallel striatal and hippocampal systems for landmarks and boundaries in spatial memory. Proceedings of the National Academy of Sciences, U.S.A., 105, 5915-5920.

Epstein, R. (2008). Parahippocampal and retrosplenial contributions to human spatial navigation. Trends in Cognitive Sciences, 12, 388-396.

Epstein, R., Harris, A., Stanley, D., \& Kanwisher, N. (1999). The parahippocampal place area: Recognition, navigation, or encoding? Neuron, 23, 115-125.

Epstein, R., \& Kanwisher, N. (1998). A cortical representation of the local visual environment. Nature, 392, 598-601.

Epstein, R. A., Higgins, J. S., \& Thompson-Schill, S. L. (2005). Learning places from views: Variation in scene processing as a function of experience and navigational ability. Journal of Cognitive Neuroscience, 17, 73-83.

Etchamendy, N., \& Bohbot, V. D. (2007). Spontaneous navigational strategies and performance in the virtual town. Hippocampus, 17, 595-599.

Fox, M. D., \& Raichle, M. E. (2007). Spontaneous fluctuations in brain activity observed with functional magnetic resonance imaging. Nature Reviews Neuroscience, 8, 700-711.

Griswold, M. A., Jakob, P. M., Heidemann, R. M., Nittka, M., Jellus, V., Wang, J., et al. (2002). Generalized autocalibrating partially parallel acquisitions (GRAPPA). Magnetic Resonance in Medicine, 47, 1202-1210.

Hartley, T., Maguire, E. A., Spiers, H. J., \& Burgess, N. (2003). The well-worn route and the path less traveled: Distinct neural bases of route following and wayfinding in humans. Neuron, 37, 877-888.

Hasson, U., Nusbaum, H. C., \& Small, S. L. (2009). Task-dependent organization of brain regions active during rest. Proceedings of the National Academy of Sciences, U.S.A., 106, 10841-10846.

Hayasaka, S., \& Nichols, T. E. (2003). Validating cluster size inference: Random field and permutation methods. Neuroimage, 20, 2343-2356. 
Hegarty, M., Montello, D., Richardson, A., Ishikawa, T., \& Lovelace, K. (2006). Spatial abilities at different scales: Individual differences in aptitude-test performance and spatial-layout learning. Intelligence, 34, 151-176.

Hegarty, M., Richardson, A., Montello, D., \& Lovelace, K. (2002). Development of a self-report measure of environmental spatial ability. Intelligence, 30, 425-447.

Iaria, G., Chen, J.-K., Guariglia, C., Ptito, A., \& Petrides, M. (2007). Retrosplenial and hippocampal brain regions in human navigation: Complementary functional contributions to the formation and use of cognitive maps. European Journal of Neuroscience, 25, 890-899.

Iaria, G., Petrides, M., Dagher, A., Pike, B., \& Bohbot, V. D. (2003). Cognitive strategies dependent on the hippocampus and caudate nucleus in human navigation: Variability and change with practice. Journal of Neuroscience, 23, 5945-5952.

Janzen, G., \& Jansen, C. (2010). A neural wayfinding mechanism adjusts for ambiguous landmark information. Neuroimage, 52, 364-370.

Janzen, G., Jansen, C., \& van Turennout, M. (2008). Memory consolidation of landmarks in good navigators. Hippocampus, 18, 40-47.

Janzen, G., \& van Turennout, M. (2004). Selective neural representation of objects relevant for navigation. Nature Neuroscience, 7, 673-677.

Janzen, G., Wagensveld, B., \& van Turennout, M. (2007). Neural representation of navigational relevance is rapidly induced and long lasting. Cerebral Cortex, 17, 975-981.

Janzen, G., \& Weststeijn, C. G. (2007). Neural representation of object location and route direction: An event-related fMRI study. Brain Research, 1165, 116-125.

Kato, Y., \& Takeuchi, Y. (2003). Individual differences in wayfinding strategies. Journal of Environmental Psychology, 23, 171-188.

Maguire, E. A., Frackowiak, R. S., \& Frith, C. D. (1996). Learning to find your way: A role for the human hippocampal formation. Proceedings of the Royal Society of London, Series B, Biological Sciences, 263, 1745-1750.

Maguire, E. A., Frith, C. D., Burgess, N., Donnett, J. G., \& O'Keefe, J. (1998). Knowing where things are parahippocampal involvement in encoding object locations in virtual large-scale space. Journal of Cognitive Neuroscience, 10, 61-76.

McDonald, R. J., \& White, N. M. (1994). Parallel information processing in the water maze: Evidence for independent memory systems involving dorsal striatum and hippocampus. Behavioral and Neural Biology, 61, 260-270.

Miall, R. C., \& Robertson, E. M. (2006). Functional imaging:
Is the resting brain resting? Current Biology, 16, R998-R1000.

O'Keefe, J., \& Nadel, L. (1978). The bippocampus as a cognitive map. Oxford: Clarendon.

Packard, M. G., \& McGaugh, J. L. (1996). Inactivation of hippocampus or caudate nucleus with lidocaine differentially affects expression of place and response learning. Neurobiology of Learning and Memory, 65, 65-72.

Prestopnik, J., \& Roskos-Ewoldsen, B. (2000). The relations among wayfinding strategy use, sense of direction, sex, familiarity, and wayfinding ability. Journal of Environmental Psychology, 20, 177-191.

Schinazi, V. R., \& Epstein, R. A. (2010). Neural correlates of real-world route learning. Neuroimage, 53, 725-735.

Shelton, A. L., \& Gabrieli, J. D. E. (2002). Neural correlates of encoding space from route and survey perspectives. Journal of Neuroscience, 22, 2711-2717.

Tambini, A., Ketz, N., \& Davachi, L. (2010). Enhanced brain correlations during rest are related to memory for recent experiences. Neuron, 65, 280-290.

Tzourio-Mazoyer, N., Landeau, B., Papathanassiou, D., Crivello, F., Etard, O., Delcroix, N., et al. (2002). Automated anatomical labeling of activations in SPM using a macroscopic anatomical parcellation of the MNI MRI single-subject brain. Neuroimage, 15, 273-289.

van Kesteren, M. T. R., Fernández, G., Norris, D. G., \& Hermans, E. J. (2010). Persistent schema-dependent hippocampal-neocortical connectivity during memory encoding and postencoding rest in humans. Proceedings of the National Academy of Sciences, U.S.A., 107, 7550-7555.

Voermans, N. C., Petersson, K. M., Daudey, L., Weber, B., Van Spaendonck, K. P., Kremer, H. P. H., et al. (2004). Interaction between the human hippocampus and the caudate nucleus during route recognition. Neuron, 43, 427-435.

Waites, A. B., Stanislavsky, A., Abbott, D. F., \& Jackson, G. D (2005). Effect of prior cognitive state on resting state networks measured with functional connectivity. Human Brain Mapping, 24, 59-68.

Weniger, G., Siemerkus, J., Schmidt-Samoa, C., Mehlitz, M., Baudewig, J., Dechent, P., et al. (2010). The human parahippocampal cortex subserves egocentric spatial learning during navigation in a virtual maze. Neurobiology of Learning and Memory, 93, 46-55.

Wolbers, T., \& Büchel, C. (2005). Dissociable retrosplenial and hippocampal contributions to successful formation of survey representations. Journal of Neuroscience, 25, $3333-3340$ 